\title{
The integration of genomics into clinical ophthalmic services in the UK
}

\author{
G. C. Black ${ }^{1,2,3} \cdot$ C. MacEwen ${ }^{4,5} \cdot$ A. J. Lotery ${ }^{6,7}$
}

Received: 31 October 2019 / Accepted: 11 November 2019 / Published online: 25 November 2019

(c) The Royal College of Ophthalmologists 2019

\section{Introduction}

Over the past 3 decades a series of discoveries in the specialty of ophthalmology have been central to furthering the understanding of genetic disease. However, currently ophthalmic genomics is seen as a niche area practised by a small number of highly trained experts; but things are changing. As part of the NHS England Long Term plan there is now a major initiative to develop personalised approaches to medicine with delivery of 500,000 individuals offered whole-genome sequencing (WGS) by 2023 [1]. As such, genomic testing in ophthalmology is due to change significantly with the introduction of a national directory of approved genetic tests, three appointed laboratory providers of specialised ophthalmic genomic testing and importantly, centralised funding for most rare disease testing. The other three home nations are also at advanced stages of planning in this area. Therefore, now it is an opportune time to consider how to integrate genomics into mainstream ophthalmic practice.

\section{G. C. Black}

graeme.black@manchester.ac.uk

1 Division of Evolution and Genomic Sciences, School of Biological Sciences, Faculty of Biology, Medicine and Health, Manchester Academic Health Science Centre, University of Manchester, Manchester, UK

2 NW Genomic Laboratory Hub, St Mary's Hospital, Manchester Centre for Genomic Medicine, Manchester Academic Health Sciences Centre, Manchester University NHS Foundation Trust, Manchester, UK

3 Manchester Centre for Genomic Medicine, Central Manchester University Hospitals NHS Foundation Trust, Manchester

\section{A wide range of discoveries-from rare to common}

It is perhaps ironic that genetic discoveries within ophthalmology have become so frequent and so numerous that we downplay their clinical significance and treat them as commonplace. From rare disease to common, from cancer to neuro-degeneration, they are quite the reverse.

Retinoblastoma, the paradigm for understanding tumour suppressor gene mutation, is a rare, familial and, let us not forget, lethal cancer-predisposing condition. It should not be underestimated that, for most of those provided with an early genetic diagnosis and specialist therapeutic intervention, retinoblastoma has become curable in most Western countries.

Inherited retinal diseases are now known to be a group of staggering breadth and heterogeneity. The sheer length of the list of gene discoveries makes the eyes, of even the most hardened super-specialist, glaze over. Recent groundbreaking publications show that successful, targeted interventions are, at last, becoming available. Treatment trials of potential therapies ranging from conventional drugs (for Leber hereditary optic neuropathy) to gene replacements (CHM, RPE65); and antisense technologies (for Leber congenital amaurosis) represent an extraordinary potential

Academic Health Science Centre, St. Mary's Hospital, Manchester, UK

4 Academy of Medical Royal Colleges, 10 Dallington St, London, UK

5 Ophthalmology Department, University of Dundee, Ninewells Hospital, Dundee, UK

6 Clinical and Experimental Sciences, Faculty of Medicine, University of Southampton, University Hospital Southampton, Tremona Road, Southampton, England, UK

7 Southampton Eye Unit, University Hospital Southampton NHS Foundation Trust, University Hospital Southampton, Southampton, England, UK 
turnaround for groups of patients who were told, until recently, that there is 'nothing we can do' $[2,3]$.

There will be those who argue that genetic discoveries will never have true impact until they apply to conditions affecting more than 2 in 10,000 individuals, a frequency below which a disease is defined as rare-although 17 million individuals, the number of patients in the UK with rare diseases, might take exception to this view. However genetics is directly relevant to our commonest diseases. Progress in unravelling the genetic aetiology of agerelated macular disease, the commonest reason for visual impairment registration in the UK, is now far advanced and probably ahead of any other common/'polygenic'/ complex genetic disorders. This has shown how complement dysregulation results in localised macular inflammation, which has now started to lead to novel treatment approaches.

\section{Important yet often undervalued. Why?}

Currently these discoveries feel beyond the reach of, and more crucially of little help to, the mainstream ophthalmologist. To the busy clinician genetic advances have perhaps reasonably carried a strong scent of 'So What?'. As a consequence, few patients with genetic disease receive a molecular diagnosis, few clinicians know patients who have received a gene-based diagnosis, and fewer still will, themselves, have ever ordered a genetic test. Yet it is likely that all will know patients affected by genetic eye disease. Why the disconnect? In large part, this has been due to the difficulty in reliably getting genetic diagnoses via molecular testing. Prior to this year, UK genetic testing budgets were held outside of ophthalmology and this often resulted in case by case assessments to justify testing. This required specialist knowledge and made it challenging for many to order and fund genomic testing, resulting in little or no testing within the context of the NHS system. This is now changing (Fig. 1).

Two things are happening that should make us stand up and take note.

The first is a rapid change in genomic diagnostic technologies, with evidence that they are cost effective. Until very recently, technological limitations meant that genetic testing was possible for only a tiny minority of rare conditions, at high cost. However, the ability to sequence large portions of an individual's DNA at the same time ('massively parallel sequencing') — indeed to undertake WGSmeans that, for rare diseases, as well as for many tumour types, molecular analysis is now possible at a price hitherto unimaginable (the sequencing of a whole genome is currently estimated to cost about $£ 1000$ ).

The second is a change to health budgets and a reorganisation of genetic services. NHS England has undertaken a landmark revamp of the supporting infrastructure and the other home nations are similarly engaged in providing new services with variations in funding and hub models [4]. There are three key elements in England that are listed in Fig. 1. They include centralised funding for rare disease testing, a novel genomic test directory (Fig. 2) and the creation of seven laboratories ('Genomic Laboratory Hubs') to provide support for NHS testing-of which three will undertake testing of ophthalmic genetic conditions. As a consequence, molecular genetic testing is both possible and affordable within structures in place to deliver it. It is fair to say that this creates a unique and national genomic medicine service that offers considerable opportunity. The education of all health care staff in genomics and set up of clinical pathways regionally will secure the future of this benefit to patients.
Fig. 1 National changes in Genomic Medicine and the creation of a National Genomic Medicine Service

\section{Key changes to the structure of Genomic Medicine Delivery in England that will impact on Ophthalmology:}

1. Centralised funding for rare disease testing. Any patient for whom a test is indicated is eligible to be tested. The ordering clinician does not need to pay for the test, which is budgeted centrally by NHSE.

Alongside this are two other changes that will facilitate ordering

2. A directory of genetic tests that defines a comprehensive range of conditions for which specific tests are now available.

3. The creation of three testing laboratories for ophthalmic genetics, allowing the development of clinical (multidisciplinary management and reporting) structures to support test ordering and interpretation. 
Fig. 2 Diagnostic testing for rare ophthalmic disorders using genomic approaches including gene panels, whole exome and whole genome sequencing

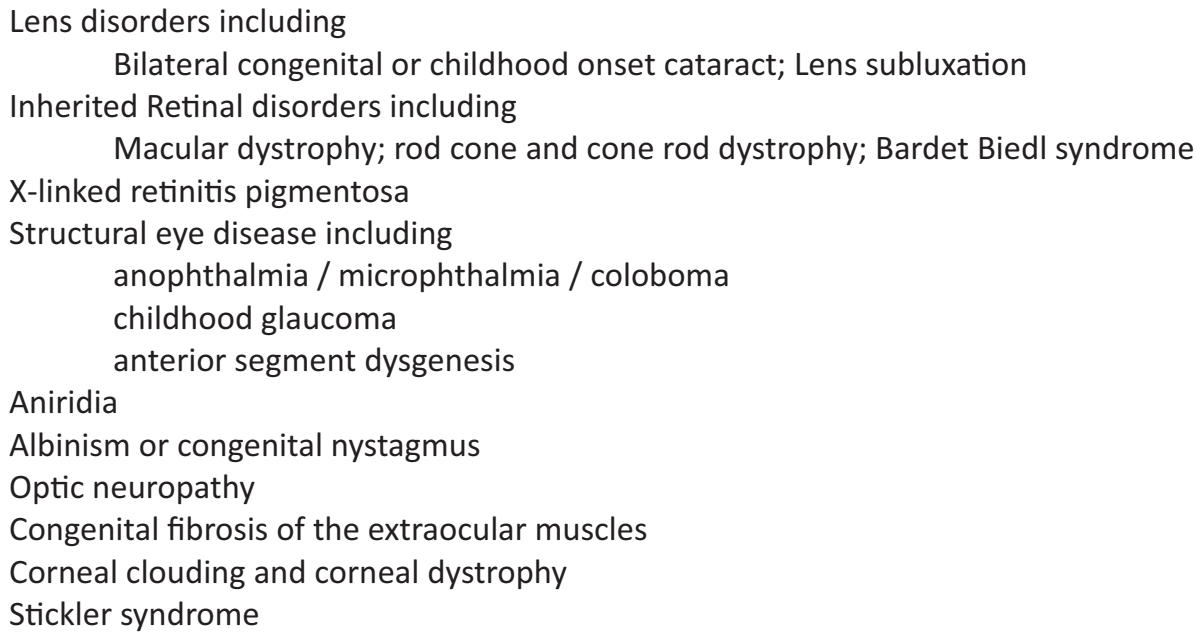

\section{Why does this matter-and to whom?}

First, this matters to patients. Patients with rare diseases seek testing to provide closure and complete their search for a diagnosis-ending their so-called 'diagnostic odyssey'. Patients correctly see this helping with clinical decisionmaking, better understanding of their prognosis and defining if there are any current, or future, therapeutic options available to them. However a clear diagnosis also helps with coping, adjustment, understanding family risks and emotional preparation for family members.

Second, this matters to clinicians and providers of health care. Efficient testing can save both the time and costs that result from the ordering of multiple expensive (usually negative) and consecutive diagnostic investigations, a situation that is common for patients with complex/rare disease. The consequence of these inefficiencies, and what is often acutely felt by patients with rare disorders, is the exacerbation of disease burden through delayed diagnosis, inaccurate management, inappropriate appointments and incorrect referral into specialist care.

Lastly, it is likely that this will improve equity of access. Since specialist care has often developed in a piecemeal manner based upon an individual clinician's special interests, those with rare diseases are more likely to be diagnosed if they see-or live close to-a clinician who happens to recognise that condition or to have seen it before. That is, access to specialist care has a strong dependence on luck. Removing the physical barrier that resulted from an inability to provide and pay for genomic testing has the potential to ensure that any patient who can benefit from such testing (i.e. get a diagnosis) is able to access it.

\section{Now is the time to get involved}

The necessary structures have been developed to make genomic testing more widely available.

In the first instance, this will make existing clinical pathways smoother, particularly for individuals with inherited paediatric disorders (e.g. congenital cataract; early-onset nystagmus, lens dislocation, macular dystrophy, optic neuropathy), adult retinal conditions and for those with rare diseases, genomic testing is likely to be performed earlier in the care pathway and become an integral part of diagnosis and management.

However, the stated aim is to widen access to genetic testing to more patients by encouraging its use by a broader group of clinicians that sit outside those few experts currently 'in the know'. It is important to remember that all ophthalmic clinicians see patients with rare diseases as well as common conditions with genetic causes or influences. Indeed, most rare ophthalmic conditions are first seen by general ophthalmologists. Thus, it is vital that all ophthalmologists are aware of and become part of the roll out of the new system so that there will be accelerated diagnosis, improved access to novel therapies and streamlined care.

It will, therefore, be critical that those undertaking the testing are provided with the clearest understanding of firstly, how such tests may be ordered, with access to appropriate training, and secondly how they may return results to patients safely and with confidence. Education and training packages, as well as defined pathways of care, that have the multidisciplinary support of clinical geneticists and genomic counsellors (infrastructure support) are being developed to this end. The development of local, regional and national clinical networks, using precise multidisciplinary care approaches to work within the new 
genomic medical service is underway. It is essential that all clinicians are aware of the existence of this infrastructure and that support is available to deliver this care to the patients who will benefit from it.

However there is a second imperative that sits within the clinical specialties. Genomics has always been seen as sitting somewhat aloof, making it easy for the generalist to ignore. As this changes and it moves closer to the heart of mainstream medicine in all specialities, this approach will become unjustifiable. The shift in emphasis-the so-called 'mainstreaming' of genomic medicine-will require supporting a motivated ophthalmic workforce to truly integrate genomics into ophthalmology. The national changes in genomic testing represent a great opportunity to provide better integrated care for our patients, which we must embrace as ophthalmologists. Resources already exist to improve our understanding of genomics and the Royal College of Ophthalmologists is currently reviewing how it can provide more genomic resources and training for its members $[5,6]$.

\section{Compliance with ethical standards}

Conflict of interest AJL is Chair of the Genomics Working Group at the Royal College of Ophthalmologists. GCB is Genomics Clinical Lead for the Royal College of Ophthalmologists. CME is Chair of the Academy of Medical Royal Colleges.

Publisher's note Springer Nature remains neutral with regard to jurisdictional claims in published maps and institutional affiliations.

\section{References}

1. NHS longterm plan. https://www.longtermplan.nhs.uk/wp-content/ uploads/2019/08/nhs-long-term-plan-version-1.2.pdf. (2019).

2. Jurkute N, Harvey J, Yu-Wai-Man P. Treatment strategies for Leber hereditary optic neuropathy. Curr Opin Neurol. 2019;32:99-104.

3. Sahel JA, Dalkara D. Gene therapy for retinal dystrophy. Nat Med. 2019;25:198-9. https://doi.org/10.1038/s41591-019-0346-1.

4. NHS genomic medicine service. https://www.england.nhs.uk/ genomics/genomic-laboratory-hubs/.

5. UK eye genetic group. https://ukegg.com/education/\#genomics

6. NHS England-genomics education materials. https://www.engla nd.nhs.uk/genomics/. 